\title{
A novel index for assessing treatment plan quality in stereotactic radiosurgery
}

\author{
Alexis Dimitriadis, PhD, and lan Paddick, MSc \\ Queen Square Radiosurgery Centre, National Hospital for Neurology and Neurosurgery, London, United Kingdom
}

OBJECTIVE Stereotactic radiosurgery (SRS) is characterized by high levels of conformity and steep dose gradients from the periphery of the target to surrounding tissue. Clinical studies have backed up the importance of these factors through evidence of symptomatic complications. Available data suggest that there are threshold doses above which the risk of symptomatic radionecrosis increases with the volume irradiated. Therefore, radiosurgical treatment plans should be optimized by minimizing dose to the surrounding tissue while maximizing dose to the target volume. Several metrics have been proposed to quantify radiosurgical plan quality, but all present certain weaknesses. To overcome limitations of the currently used metrics, a novel metric is proposed, the efficiency index $\left(\eta_{50 \%}\right)$, which is based on the principle of calculating integral doses: $\eta_{50 \%}=$ integral dose $\mathrm{TV}_{\mathrm{TV}}$ /integral dose $\mathrm{PIV}_{\mathrm{P} 0 \%}$.

METHODS The value of $\eta_{50 \%}$ can be easily calculated by dividing the integral dose (mean dose $\times$ volume) to the target volume (TV) by the integral dose to the volume of $50 \%$ of the prescription isodose (PIV50\%). Alternatively, differential dose-volume histograms (DVHs) of the TV and PIV50\% can be used. The resulting $\eta_{50 \%}$ value is effectively the proportion of energy within the PIV50\% that falls into the target. This value has theoretical limits of 0 and 1 , with 1 being perfect. The index combines conformity, gradient, and mean dose to the target into a single value. The value of $\eta_{50 \%}$ was retrospectively calculated for 100 clinical SRS plans.

RESULTS The value of $\eta_{50 \%}$ for the 100 clinical SRS plans ranged from $37.7 \%$ to $58.0 \%$ with a mean value of $49.0 \%$. This study also showed that the same principles used for the calculation of $\eta_{50 \%}$ can be adapted to produce an index suitable for multiple-target plans $\left(G \eta_{12 G y}\right)$. Furthermore, the authors present another adaptation of the index that may play a role in plan optimization by calculating and minimizing the proportion of energy delivered to surrounding organs at risk $\left(\mathrm{OAR} \eta_{50 \%}\right)$.

CONCLUSIONS The proposed efficiency index is a novel approach in quantifying plan quality by combining conformity, gradient, and mean dose into a single value. It quantifies the ratio of the dose "doing good" versus the dose "doing harm," and its adaptations can be used for multiple-target plan optimization and OAR sparing.

https://thejns.org/doi/abs/10.3171/2018.7.GKS18694

KEYWORDS radiotherapy; conformity; gradient; index; plan quality; stereotactic radiosurgery

$\mathrm{S}$ TEREOTACTIC radiosurgery (SRS) is defined as a high dose of radiation, most commonly prescribed to an intracranial target. In intracranial treatments, the target is often surrounded by healthy brain, which is usually the primary organ at risk (OAR). In these treatments, it is essential to achieve a steep dose falloff from the periphery of the target to normal tissue in order to minimize normal tissue toxicity and resulting complications.

Several studies have demonstrated the necessity for reducing volumes of high doses outside the target volume (TV; achieved by steep dose gradients) through evidence from clinical outcomes. An early study by Flickinger et al. showed a strong correlation between symptomatic radiation necrosis and a 12-Gy isodose volume in patients treated with radiosurgery for arteriovenous malformation (AVM) ${ }^{11}$ The observations of Korytko et al. added to and expanded the knowledge on postradiosurgical toxicity, as the same correlation was also observed for non-AVM patients. ${ }^{17}$ In recent years, the evidence has grown, further supporting the importance of limiting the dose received by surrounding brain tissue. ${ }^{6,20,27}$ The available data suggest that there are threshold doses above which the risk of symptomatic radionecrosis increases with the volume irradiated. Therefore, it makes sense to concentrate on limit-

ABBREVIATIONS AUC = area under the curve; CSF = cerebrospinal fluid; $\mathrm{DVH}=$ dose-volume histogram; $\mathrm{GI}=$ gradient index; $\mathrm{G} \eta=$ global efficiency index; $\eta_{50 \%}=$ efficiency index; $\mathrm{OAR}=$ organ at risk; $\mathrm{OAR} \eta_{50 \%}=\mathrm{OAR}$ efficiency index; $\mathrm{PCI}=$ Paddick conformity index; $\mathrm{PIV}=$ prescription isodose volume; $\mathrm{PIV} 50 \%=$ volume of half the prescription isodose; PTV = planning target volume; SRS = stereotactic radiosurgery; TCP = tumor control probability; TV = target volume.

SUBMITTED May 31, 2018. ACCEPTED July 26, 2018.

INCLUDE WHEN CITING DOI: 10.3171/2018.7.GKS18694. 
ing doses above these thresholds and ignoring lower doses that may be clinically irrelevant.

The International Commission on Radiation Units \& Measurements (ICRU) has recently published Report 91, "Prescribing, recording, and reporting of stereotactic treatments with small photon beams," in which the importance of the dose falloff is highlighted: "This [dose falloff] could be represented again by the volume of a specified isodose (e.g., $\mathrm{V}_{12 \mathrm{~Gy}}$-volume receiving dose larger than or equal to $12 \mathrm{~Gy}$ ) but, despite the simplicity and biologic rationale, this has not gained widespread acceptance because it does not lend itself to comparing or summarizing plan quality between targets of different sizes treated to different doses." 15

Several indices have been proposed over the years to quantify levels of conformity and/or dose falloff in radiotherapy plans. ${ }^{10,25}$ Some involve cumbersome calculation methods and have proven difficult to routinely incorporate into radiosurgical practice, ${ }^{28,32}$ and some favor dose homogeneity inside the target, ${ }^{2}$ a controversial parameter that could be considered inappropriate for radiosurgery given its detrimental effect on dose falloff outside the target. ${ }^{34}$ For an index to be adopted, it is important for it to be intuitive, easily calculated, and aligned with the radiosurgery planning philosophy.

The gradient index (GI), ${ }^{24}$ defined as the ratio of the volume of half the prescription isodose (PIV50\%) to the prescription isodose volume (PIV), has been extensively used over the last decade. The GI usually complements the conformity index ${ }^{23}$ to quantify the dose falloff for a plan of a given conformity. It remains the most commonly used metric, serving as a practical volumetric assessment of dose falloff. It was originally proposed that a GI $\leq 3.0$ could be achieved and should be aimed for in the treatment of single targets. An empirically derived GI value of 2.4 is near perfect. Upon formulating the GI, the authors identified its limitation in being unsuitable for comparing plans of incongruent conformity. Moreover, while the metric is volumetric and quantifies the overall falloff between two isodose volumes, it does not account for the intermediate doses between the two isodoses.

In order to overcome this limitation, Thomas et al. ${ }^{30}$ proposed the use of the area under the curve (AUC) metric, previously used for predicting toxicity in genitourinary and gastrointestinal treatments, ${ }^{16,31,33}$ as an alternative index for comparative plan evaluation in SRS. The AUC metric is the integral area under the dose-volume histogram (DVH) between the PIV50\% and the PIV. This metric provides a useful dose-volume product that quantifies dose falloff outside the target in the region of moderate to high dose, which is arguably more descriptive than the ratio of two isodose volumes. However, this metric suffers from dependence on prescription dose and does not account for conformity (dose spillage). As a result, only the dose outside the PIV is scored, not the dose outside the target itself. This suggests the need for alternative quality metrics for plan optimization that combine the quality of conformity and gradient.

In this paper, we propose a plan quality index that is aligned with the planning principles of radiosurgery and is suitable for assessing both conformity and gradient in a single value, without prioritizing one over the other. We show that this index can be adapted to allow plan quality assessments in multiple-target plans and for OAR sparing. The method of calculation and the range of clinically acceptable plans are presented as proof of principle for the indices proposed.

\section{Methods}

Efficiency is classically defined as the ratio of useful output energy over the total input energy used by a system. When applying the concept of efficiency to the quality of a radiosurgery treatment plan, "useful energy" is interpreted as that delivered to the TV. With the approximation that tissue has the density of water, the product of dose and volume equals energy in millijoules. In terms of the total energy used, evidence suggests that lower isodose volumes can be disregarded as they are clinically irrelevant in the prediction of symptomatic radionecrosis. It is, therefore, appropriate to adopt a lower cutoff value above which the efficiency of a treatment plan can be assessed. For continuity with previously used indices, we adopted half of the prescription dose as the threshold dose for the analysis of plans with single targets. For prescription doses of 24 Gy, this half prescription isodose corresponds to the 12Gy volume.

\section{Efficiency Index in Clinical SRS Plans}

Based on the principles described above, a novel metric, the efficiency index $\left(\eta_{50 \%}\right)$, is proposed to overcome the limitations of previously used indices. The index is calculated as the ratio of the integral dose of TV to the integral dose of PIV50\%:

$$
\eta_{50 \%}=\frac{\text { Useful Energy }}{\text { Total Energy }}=\frac{\text { Integral Dose }_{\mathrm{TV}}}{\text { Integral Dose }}=\frac{\int_{\mathrm{PIV} 50 \%}^{\mathrm{Dmax}} \mathrm{TV} \delta \text { dose }}{\int_{\mathrm{PIV} 50 \%}^{\mathrm{Dmax}} \mathrm{V} \delta \text { dose }}
$$

where $\mathrm{D}_{\min }$ is the minimum dose to the target, $\mathrm{D}_{\max }$ is the maximum dose to the target, TV is the target volume, and PIV50\% is the volume occupied by $50 \%$ of the prescription dose.

In order to calculate $\eta_{50 \%}$ for a single-target treatment plan, one needs to calculate the integral dose to the TV and the integral dose of PIV50\%. The simplest method for doing this is to multiply the volume of the structure by the mean dose to the structure as shown in equation 2:

$$
\text { Integral Dose } \mathrm{DV}_{\mathrm{TV}}=\text { Mean Dose }_{\mathrm{TV}} \times \text { Volume }_{\mathrm{TV}} \text {. }
$$

A graphic representation of the proposed index for a single-target treatment plan is shown in Fig. 1.

Most treatment planning systems (TPSs) have the option of converting an isodose line volume to a structure, which facilitates the calculation of integral dose and $\eta_{50 \%}$. When this is not an option (e.g., in Leksell GammaPlan, Elekta AB), $\eta_{50 \%}$ can be calculated by producing two differential DVHs from the TPS: one for the target structure and one for a structure or region encompassing the isodose line of the threshold dose adopted (PIV50\%). Each differential DVH is exported in numerical format and used to calculate the AUC within the limits shown in equation 1. The calculated area under the DVH curve of the TV struc- 
ture is the numerator, and the area under the DVH curve of the PIV50\% structure is the denominator. The value generated by equation 1 is the ratio of energy deposited inside the target over the total energy deposited within PIV50\%. This value has theoretical limits between 0 and 1 , with 1 being the perfect score.

As the dose gradient outside the target becomes steeper, $\eta_{50 \%}$ increases due to a decrease in the denominator value. Moreover, $\eta_{50 \%}$ also increases with improvements in conformity, which increase the numerator value (with improved coverage) and decrease the denominator value (with improved selectivity). Furthermore, an increase in the mean dose deposited within the TV due to hotter areas (regions of high dose) will also be quantified as an increase in $\eta_{50 \%}$ due to an increase in the numerator. Finally, volumes are proportionally weighted by the dose received because energy is volume multiplied by dose. Effectively, the efficiency index $\left(\eta_{50 \%}\right)$ is a single value that quantifies plan quality and accounts for conformity, gradient, and a high mean dose to the target.

In order to investigate the variation of $\eta_{50 \%}$ with variations in conventional plan quality, the value of $\eta_{50 \%}$ was retrospectively calculated for 100 clinical intracranial radiosurgery treatments planned on Leksell GammaPlan. These plans were for a range of indications and prescription doses with a TV range of $0.1-13.2 \mathrm{~cm}^{3}$ with a mean value of $2.8 \mathrm{~cm}^{3}$. The calculated $\eta_{50 \%}$ values were compared to the commonly used Paddick conformity index (PCI) and GI. The tested hypothesis was that gradient and conformity improvements would correlate with an increase in the value of $\eta_{50 \%}$.

\section{Efficiency Index in Multiple-Target Plans}

The new index was adapted to quantify plan quality in cases with multiple targets that were close to each other. Such situations are becoming more common in radiosurgery as the modality is replacing whole-brain radiotherapy for the management of metastatic brain disease..$^{18}$ Quantifying dose falloff in multiple-target treatment plans can be challenging due to potential bridging of the PIV50\% for nearby targets, which can void the calculation of GI values. In order to overcome this limitation, the global efficiency index $(\mathrm{G \eta})$ is proposed to express the sum of energies deposited in the targets as a percentage of the total energy deposited in the patient. As previously mentioned, it is appropriate to apply a cutoff threshold value above which the index is calculated. As prescription isodoses may differ between targets of the same treatment plan, the $12-G y$ cutoff value is considered more appropriate than the PIV50\%. Equation 3 is the formula for calculating the $\mathrm{G} \eta_{12 \mathrm{~Gy}}$ for a plan with two targets:

$$
\mathrm{G \eta}_{12 \mathrm{~Gy}}=\frac{\text { Integral Dose }_{\mathrm{TV} 1}+\text { Integral Dose }_{\mathrm{TV} 2}}{\text { Global Integral Dose }_{12 \mathrm{~Gy}}} .
$$

In order to demonstrate the value of $\mathrm{G}_{12 \mathrm{~Gy}}$, five clinical treatment plans with multiple targets in which the calculation of GI was not possible were selected retrospectively and analyzed. The tested hypothesis was that the calculation of $\mathrm{G} \eta_{12 \mathrm{~Gy}}$ is possible even in complex conditions in which the GI cannot be calculated.
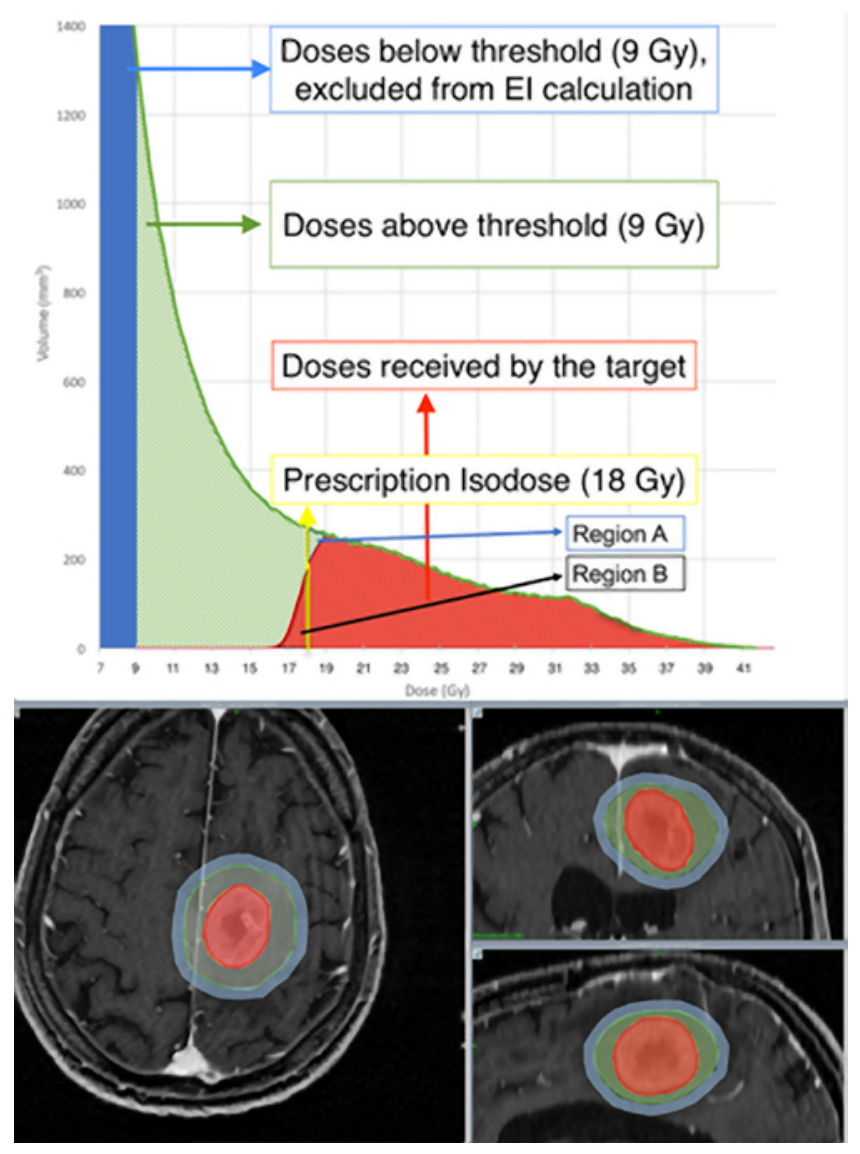

FIG. 1. Graphic representation of the proposed efficiency index (El; $\eta_{50 \%}$ ) for a single-target treatment plan. The graph (upper) shows two cumulative DVHs calculated for the plan shown (lower). The red DVH is for the target, and the green DVH is for a larger area that encompasses the target and doses above $9 \mathrm{~Gy}$. The blue area indicates regions below the threshold value of $50 \%$ of the prescription dose (outside the PIV50\%; 9 Gy in this scenario), which are excluded from the calculation of the index. The yellow line indicates the dose prescribed to the target (18 Gy). Region A represents areas where the prescription isodose spills into surrounding brain, and Region B represents areas of undercoverage by the prescription isodose. The same color scheme is used in the triplanar view shown in the plan (lower) in order to match the areas of the DVHs with areas in the treatment plan. The area under the green curve is equal to the integral dose $\mathrm{TV}_{\mathrm{TV}}$, and the area under the red curve is equal to integral dose $e_{\text {PIV } 50 \%}$. Their ratio is equal to $\eta_{50 \%}$ (equation 1 ).

\section{Efficiency Index for OARs}

The same concept of integral dose ratios can be adopted to quantify the efficiency in sparing OARs that are close to the target. Unlike $\eta_{50 \%}$, which quantifies efficiency in treating the target and where a high score is desirable, the OAR efficiency index $\left(\mathrm{OAR} \eta_{50 \%}\right)$ quantifies efficient sparing of the OAR and preferably has a low score. Equation 4 shows the formula:

$$
\mathrm{OAR \eta}_{50 \%}=\frac{\text { Integral Dose }_{\mathrm{OAR}}}{\text { Integral Dose }} \text {.0\% }
$$

It is possible to calculate $\mathrm{OAR} \eta_{50 \%}$ for any delineated structure. When multiple surrounding structures are delineated, the calculated OAR $\eta_{50 \%}$ values can be used as a guide for preferentially sparing radiosensitive surround- 

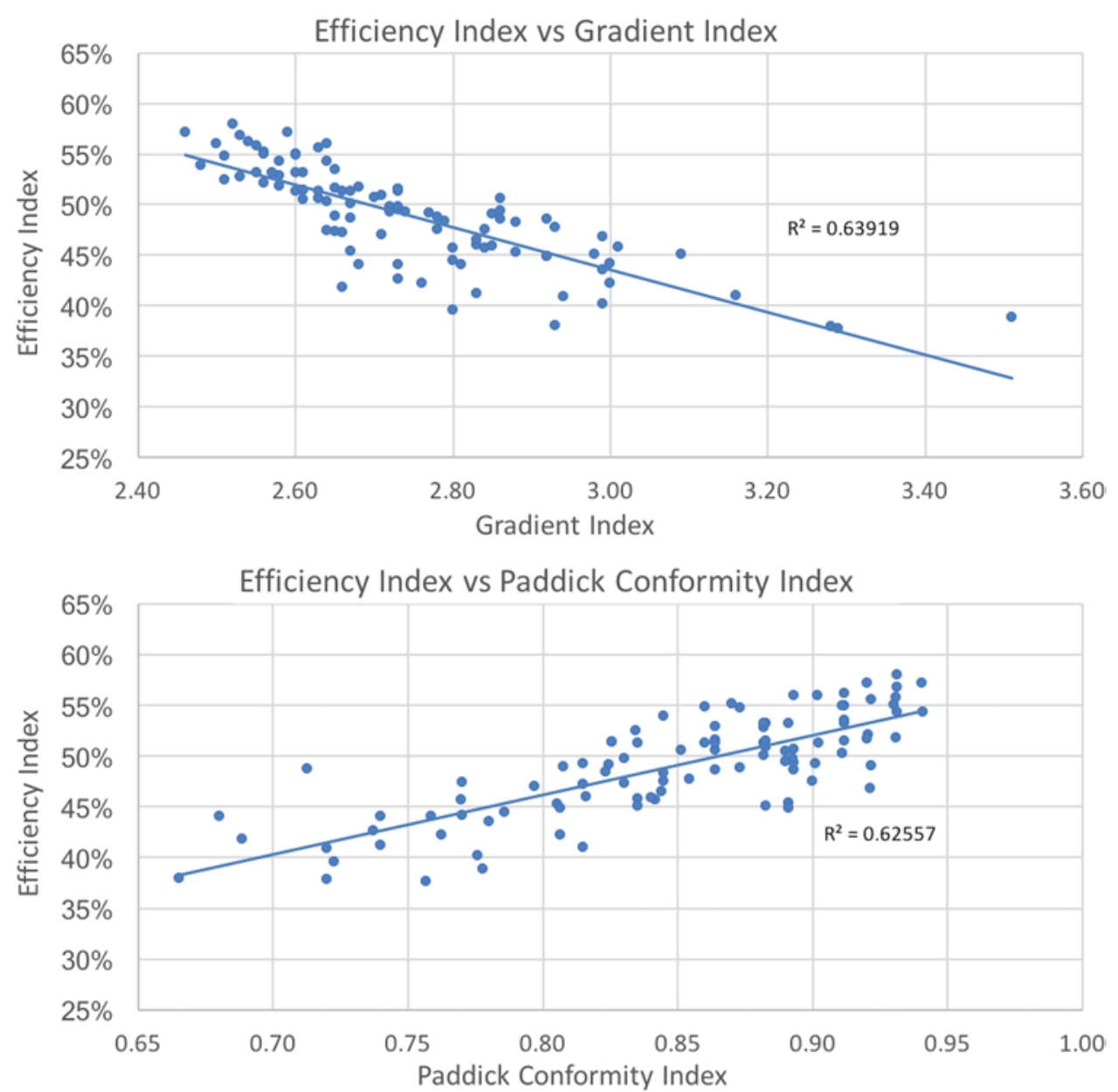

FIG. 2. Efficiency index values for the 100 single-target plans plotted against $\mathrm{GI}$ (upper) and $\mathrm{PCI}$ (lower).

ing tissue at the expense of spilling into radio-tolerant surrounding tissue. In order to investigate the potential of OAR $\eta_{50 \%}$ for such plan optimizations, a treatment plan for vestibular schwannoma was retrospectively used to contour five surrounding structures and calculate their $\mathrm{OAR} \eta_{50 \%}$ values. The five structures contoured were the cerebellum, brainstem, bone, temporal lobe, and cerebrospinal fluid (CSF). These five structures were contoured in such a way that they did not overlap, ensuring that all voxels within the PIV50\% were included in a structure. The tested hypothesis was that calculation of $\mathrm{OAR} \eta_{50 \%}$ values is possible and that, when summed together with the value of $\eta_{50 \%}$, they add up to $100 \%$, effectively describing the distribution of dose to different structures.

\section{Results}

\section{Efficiency Index in Clinical SRS Plans}

The $\eta_{50 \%}$ value for the 100 clinical SRS plans ranged from $37.7 \%$ to $58.0 \%$ with a mean value of $49.0 \%$. The results are plotted against the GI in Fig. 2 upper and PCI in Fig. 2 lower. Scores below 40\% (37.7\%-39.6\%) were seen only in small and complex targets $\left(0.1-1.0 \mathrm{~cm}^{3}\right)$ in which lower levels of conformity and/or gradient were achieved. However, for less complex targets of the same volume range, much higher scores were achieved (40.2\%-56.0\%). Scores above $56 \%(56.2 \%-58.0 \%)$ were seen in plans with higher levels of conformity and gradient for a larger target volume range $\left(2.3-5.2 \mathrm{~cm}^{3}\right)$, although the scores for the five largest targets in the series $\left(9.5-13.5 \mathrm{~cm}^{3}\right)$ ranged from $48.9 \%$ to $55.6 \%$.

\section{Efficiency Index in Multiple-Target Plans}

The treatment details and calculated $\mathrm{G}_{12 \mathrm{~Gy}}$ values for the five treatment plans analyzed are shown in Table 1. The $\mathrm{G}_{12 \mathrm{~Gy}}$ values ranged from $44.0 \%$ to $51.2 \%$. Threedimensional image sections for case 5 are shown in Fig. 3.

\section{Efficiency Index for OARs}

The plan for the selected vestibular schwannoma case shown in Fig. 4 yielded an $\eta_{50 \%}$ value of $50.1 \%$. The $\mathrm{OAR} \eta_{50 \%}$ values for the brainstem, cerebellum, bone, temporal lobe, and CSF were calculated to be $14.9 \%, 10.1 \%$, $10.0 \%, 7.9 \%$, and $7.0 \%$, respectively. The sum of all $\eta$ values was found to be $100.0 \%$ with a one decimal place approximation.

\section{Discussion}

The proposed efficiency indices $\left(\eta_{50 \%}, \mathrm{G} \eta_{12 \mathrm{~Gy}}\right.$, and 
TABLE 1. Plan details of five clinical cases in which a GI calculation was not possible due to overlaps of the PIV $\% 50$

\begin{tabular}{ccccc}
\hline Case No. & No. of Targets & $\mathrm{PD}(\mathrm{Gy})$ & $\mathrm{PI}(\%)$ & $\mathrm{G}_{\eta 12 \mathrm{~Gy}}(\%)$ \\
\hline 1 & 9 & $20-25$ & $48-95$ & 44.0 \\
\hline 2 & 2 & $20-25$ & $50-87$ & 51.2 \\
\hline 3 & 5 & $18-20$ & $40-88$ & 46.7 \\
\hline 4 & 4 & $18-22$ & $48-60$ & 48.2 \\
\hline 5 & 8 & $20-22$ & $44-93$ & 49.5 \\
\hline
\end{tabular}

$\mathrm{G}_{\eta_{126 y}}=$ global efficiency index with a 12-Gy cutoff; $P D=$ prescription dose; PI $=$ prescription isodose.

OAR $\eta_{50 \%}$ ) represent a novel approach in quantifying plan quality that favors internal dose escalation to the target. The most commonly used metrics quantify levels of conformity or gradient, but often the two can be in conflict. For example, high levels of conformity may be achieved at the expense of a poor gradient or vice versa. In such cases, the clinician weighs the risks, favors one objective over the other, and selects an appropriate peripheral dose often with less focus placed on the maximum dose inside the target. Thus, the intra-target dose is not currently a primary consideration in the clinical decision-making process when, arguably, it should be. The proposed efficiency index may have a role to play in plan quality assessments as it combines gradient and conformity into a single value. Moreover, it incorporates internal dose gradient as an optimization criterion that should help increase tumor control probability (TCP) while lowering normal tissue complication probability (NTCP).

Dose homogeneity within the target is a quality that has been traditionally valued in conventional radiation therapy as a large proportion of the planning target volume (PTV) consists of normal tissue. The NTCP increases with increasing hotspots in the irradiated volume (inhomogeneity) as normal tissue is thought to be sensitive to local variations in dose, especially when near the tolerance dose. When radiosurgery was conceived, originally for functional targets, the small $3 \times 5-\mathrm{mm}$ collimators used in the Gamma Knife prototype and a prescription dose at the maximum dose point made dose homogeneity irrelevant. A steep dose falloff outside the target was most sacrosanct, and inhomogeneity was an acceptable consequence. This treatment philosophy is more appealing for radiosurgery, where the vast majority of the PTV is tumor. In most cases, where no margin is applied, the PTV consists entirely of tumor.

Another argument used against inhomogeneity is the presence of "cold spots" in the tumor, thought to reduce the TCP, as every tumor cell needs to receive the prescription dose. However, one can avoid under-dosing cells in the radiosurgical target by ensuring high target coverage by the prescription dose. With regard to the presence of hotter areas in the radiosurgical target, it has been argued that they are beneficial as they increase the TCP.5,14 In theory, high doses within radiosurgical targets may be considered beneficial for most pathologies treated with SRS. For metastatic disease, high intratumoral doses are directed to central hypoxic areas of radioresistance that may improve the TCP ${ }^{1,3,8}$ for benign disease, they increase

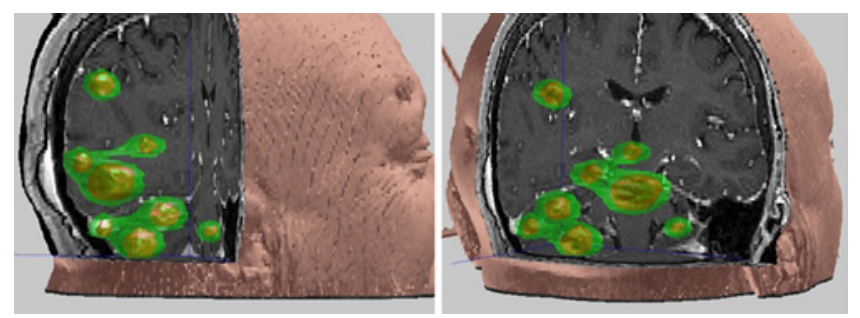

FIG. 3. Case 5 presented with eight targets. Bridging of the PIV $50 \%$ between nearby targets (green) prohibits the calculation of Gls. The $\mathrm{G} \eta_{12 \mathrm{~Gy}}$ was calculated to be $49.5 \%$.

vascular damage (aiding target shrinkage); and for secreting tumors, they may lead to faster hormone normalization. ${ }^{13,26}$ For vascular targets, they may improve favorable outcomes and possibly reduce the time to obliteration. ${ }^{9}$

As mentioned, clinical studies have consistently backed up the importance of a steep dose falloff in normal tissue surrounding the target. In order to achieve a steep dose gradient on the edge of the target, a dose gradient on the inside edge of the target is necessary. This creates a dose profile inside the target that is relatively inhomogeneous, which has been shown to improve local control. ${ }^{4}$ Recent work has verified that significant improvements in gradient can be achieved by increasing dose inhomogeneity inside the target for various platforms..$^{21,22,24,29,34}$ As a result, the majority of radiosurgery practitioners are aligned with this treatment philosophy, and the indices proposed in this

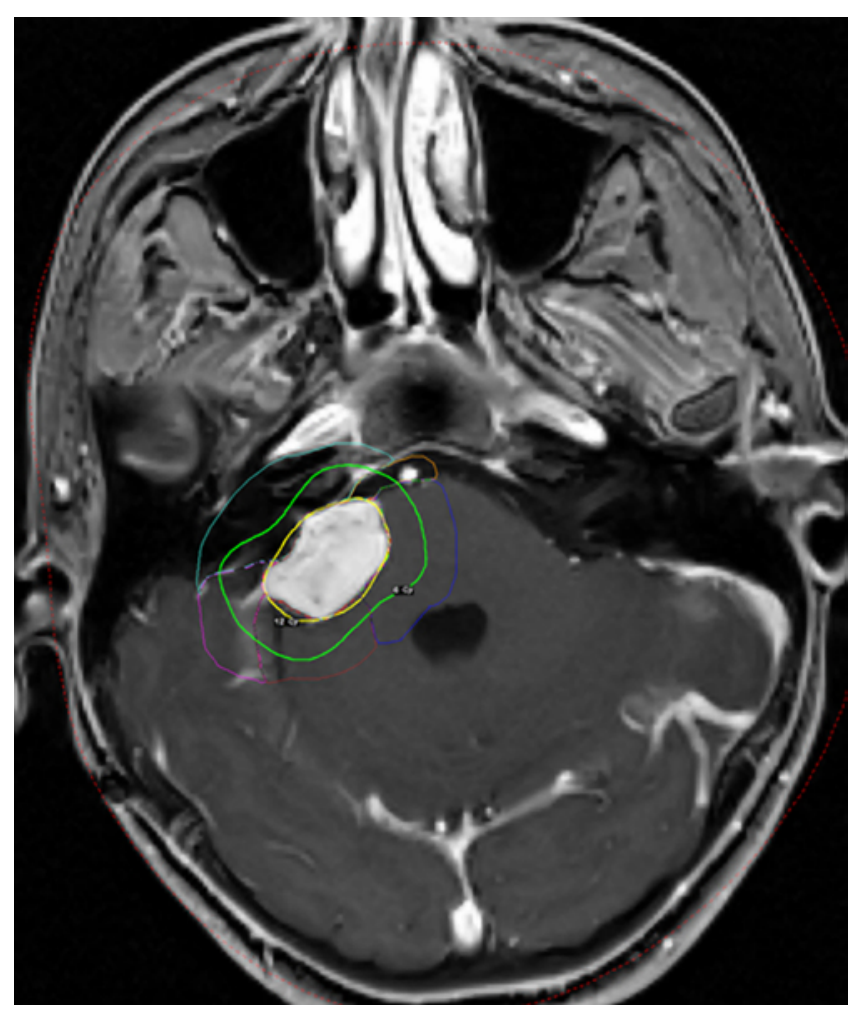

FIG. 4. Axial view of the plan for the selected vestibular schwannoma case and the six regions contoured: target volume (red), brainstem (dark blue), cerebellum (brown), temporal lobe (magenta), CSF (orange), and bone (cyan). 
study encourage the use of this philosophy. There may be scenarios in which clinical judgment weighs against higher doses in the radiosurgical target, for example, a cavernous sinus meningioma with cranial nerve involvement. In such scenarios, it may be preferable to reduce dose heterogeneity inside the target at the expense of poorer dose gradients outside, which will reduce the efficiency index score. Nevertheless, the index may still serve as a plan quality descriptor for comparative plan assessment of conformity and gradient, within the constraints of a lower mean and maximum dose in the target.

In this paper we quantified the range of $\eta_{50 \%}$ (37.7\%$58.0 \%$ ) for 100 clinical radiosurgery plans and showed correlations to improvements in both conformity and gradient. This allows the index to be used for meaningful comparisons between competing SRS plans. For targets smaller than $1 \mathrm{~cm}^{3}$, a slightly lower, but comparable, mean score $(46.4 \%$, range $37.7 \%-56.0 \%)$ for the entire population $(49.0 \%)$ was observed. The achievable scores can vary depending on the volume and complexity of the target, which impact the level of conformity and gradient achieved. Our results suggest that $\eta_{50 \%}$ values of $40.0 \%-$ $56.0 \%$ are routinely achieved in clinically acceptable Gamma Knife radiosurgery treatment plans and should be pursued.

Another two indices were formalized based on the same principles of calculation. The $\mathrm{G}_{12 \mathrm{~Gy}}$ can be used in plans with multiple targets to provide a global figure of merit for the entire plan. We demonstrated the use of this index for quantifying the quality of complex plans in which there are overlaps between the PIV $50 \%$ of nearby targets. Optimization of this index can help to lower bridging doses and increase the mean dose to all targets. Finally, $\mathrm{OAR} \eta_{50 \%}$ can provide an energy ratio value for any surrounding contoured structure. These values have the potential to be used as optimization criteria for lowering integral doses to OAR and preferentially sparing radiosensitive surrounding tissue over radio-tolerant tissue. Several studies have shown correlations between complications and dose-volume relationships in OARs., ${ }^{72,19}$ This suggests that the concept of a maximum point dose to a serial OAR is an oversimplification of its tolerance. This index favors the reduction of the dose-volume product in OARs and may help to reduce treatment complications.

As with all indices, there are limitations in the use of the proposed metrics that must be noted. If a target is severely under-covered and a large amount of the PIV50\% falls inside the target, the calculation of $\eta_{50 \%}$ may yield values higher than the quantified range presented in this paper. It is, therefore, advised to calculate the index when clinically acceptable target coverage is achieved, i.e., $\geq$ $95 \%$. In the case of multiple targets and the calculation of $\mathrm{G} \eta_{12 \mathrm{G} y}$, the cutoff threshold of $12 \mathrm{~Gy}$ was selected to align the calculation with the published evidence showing a correlation with the risk of radionecrosis. ${ }^{6,11,17,20} \mathrm{~A}$ different threshold can be chosen, but that will have an impact on the index's value. Similarly, prescribing a different dose will also affect the value. Thus, for plan comparisons to be meaningful, it is essential that competing plans use the same prescription doses and threshold values. With regard to OAR $\eta_{50 \%}$, it is possible for a structure at risk to be out- side the PIV50\% but still receive a critical dose. For example, in the vestibular schwannoma case featured in Fig. 4 , the cochlea falls outside the 6-Gy isodose line but may still require evaluation.

The proposed indices are not intended to replace current plan assessments, but rather to complement them and provide a means for improving plan quality, especially in an inverse planner setting where the index scores can be used as optimization criteria.

\section{Conclusions}

The dosimetrical concept of radiosurgery has always been to maximize dose to the target and minimize dose to normal tissue. The ability of SRS to produce such excellent results is attributable to this paradigm. A novel efficiency index, $\eta_{50 \%}$, has been proposed, which represents the intuitive ratio of useful energy to total energy. It is a volumetric approach that takes into account multiple dose levels and is more descriptive than the currently available tools for assessing radiosurgical plan quality. This index combines the qualities of conformity, gradient, and a high mean dose to the target into a single value. The proposed index excels whenever there is a compromise between these values, enabling the planner to quantify the relative proportion of the dose "doing good" versus that "doing harm." It also favors internal dose escalation to the target, and its adaptations show potential for use in multiple-target plans and planning optimization for sparing OARs. Finally, the indices presented in this study may be transferable to other forms of ablative therapy, such as high-intensity focused ultrasound and laser interstitial thermal therapy. Further work will involve studies to verify the correlation between clinical results and this proof of concept.

\section{References}

1. Abraham C, Garsa A, Badiyan SN, Drzymala R, Yang D, DeWees T, et al: Internal dose escalation is associated with increased local control for non-small cell lung cancer (NSCLC) brain metastases treated with stereotactic radiosurgery (SRS). Adv Radiat Oncol 3:146-153, 2017

2. Akpati H, Kim C, Kim B, Park T, Meek A: Unified dosimetry index (UDI): a figure of merit for ranking treatment plans. J Appl Clin Med Phys 9:2803, 2008

3. Amsbaugh M, Pan J, Yusuf MB, Dragun A, Dunlap N, Guan $\mathrm{T}$, et al: Dose-volume response relationship for brain metastases treated with frameless single-fraction linear acceleratorbased stereotactic radiosurgery. Cureus 8:e587, 2016

4. Amsbaugh MJ, Yusuf MB, Gaskins J, Dragun AE, Dunlap $\mathrm{N}$, Guan T, et al: A dose-volume response model for brain metastases treated with frameless single-fraction robotic radiosurgery: seeking to better predict response to treatment. Technol Cancer Res Treat 16:344-351, 2017

5. Andratschke N, Alheid H, Allgäuer M, Becker G, Blanck O, Boda-Heggemann J, et al: The SBRT database initiative of the German Society for Radiation Oncology (DEGRO): patterns of care and outcome analysis of stereotactic body radiotherapy (SBRT) for liver oligometastases in 474 patients with 623 metastases. BMC Cancer 18:283, 2018

6. Blonigen BJ, Steinmetz RD, Levin L, Lamba MA, Warnick $\mathrm{RE}$, Breneman JC: Irradiated volume as a predictor of brain radionecrosis after linear accelerator stereotactic radiosurgery. Int J Radiat Oncol Biol Phys 77:996-1001, 2010

7. Brisman R, Mooij R: Gamma Knife radiosurgery for trigemi- 
nal neuralgia: dose-volume histograms of the brainstem and trigeminal nerve. J Neurosurg 93 (Suppl 3): 155-158, 2000

8. Brown JM, Diehn M, Loo BW Jr: Stereotactic ablative radiotherapy should be combined with a hypoxic cell radiosensitizer. Int J Radiat Oncol Biol Phys 78:323-327, 2010

9. Ding D, Starke RM, Kano H, Mathieu D, Huang P, Kondziolka D, et al: Radiosurgery for cerebral arteriovenous malformations in A Randomized Trial of Unruptured Brain Arteriovenous Malformations (ARUBA)-eligible patients: a multicenter study. Stroke 47:342-349, 2016

10. Feuvret L, Noël G, Mazeron JJ, Bey P: Conformity index: a review. Int J Radiat Oncol Biol Phys 64:333-342, 2006

11. Flickinger JC, Kondziolka D, Lunsford LD, Kassam A, Phuong LK, Liscak R, et al: Development of a model to predict permanent symptomatic postradiosurgery injury for arteriovenous malformation patients. Int J Radiat Oncol Biol Phys 46:1143-1148, 2000

12. Flickinger JC, Lunsford LD, Kondziolka D: Dose prescription and dose-volume effects in radiosurgery. Neurosurg Clin N Am 3:51-59, 1992

13. Grant RA, Whicker M, Lleva R, Knisely JPS, Inzucchi SE, Chiang VL: Efficacy and safety of higher dose stereotactic radiosurgery for functional pituitary adenomas: a preliminary report. World Neurosurg 82:195-201, 2014

14. Guckenberger M, Klement RJ, Allgäuer M, Appold S, Dieckmann K, Ernst I, et al: Applicability of the linear-quadratic formalism for modeling local tumor control probability in high dose per fraction stereotactic body radiotherapy for early stage non-small cell lung cancer. Radiother Oncol 109:13-20, 2013

15. International Commission on Radiation Units \& Measurements: ICRU Report 91, Prescribing, Recording, and Reporting of Stereotactic Treatments with Small Photon Beams. Bethesda, MD: ICRU, 2018

16. Jani AB, Hand CM, Pelizzari CA, Roeske JC, Krauz L, Vijayakumar S: Biological-effective versus conventional dose volume histograms correlated with late genitourinary and gastrointestinal toxicity after external beam radiotherapy for prostate cancer: a matched pair analysis. BMC Cancer 3:16, 2003

17. Korytko T, Radivoyevitch T, Colussi V, Wessels BW, Pillai K, Maciunas RJ, et al: 12 Gy gamma knife radiosurgical volume is a predictor for radiation necrosis in non-AVM intracranial tumors. Int J Radiat Oncol Biol Phys 64:419-424, 2006

18. Lippitz B, Lindquist C, Paddick I, Peterson D, O'Neill K, Beaney R: Stereotactic radiosurgery in the treatment of brain metastases: the current evidence. Cancer Treat Rev 40:4859, 2014

19. Mayo C, Martel MK, Marks LB, Flickinger J, Nam J, Kirkpatrick J: Radiation dose-volume effects of optic nerves and chiasm. Int J Radiat Oncol Biol Phys 76 (3 Suppl):S28S35, 2010

20. Minniti G, Clarke E, Lanzetta G, Osti MF, Trasimeni G, Bozzao A, et al: Stereotactic radiosurgery for brain metastases: analysis of outcome and risk of brain radionecrosis. Radiat Oncol 6:48, 2011

21. Ohtakara K, Hayashi S, Hoshi H: Dose gradient analyses in Linac-based intracranial stereotactic radiosurgery using Paddick's gradient index: consideration of the optimal method for plan evaluation. J Radiat Res (Tokyo) 52:592-599, 2011

22. Ohtakara K, Hayashi S, Tanaka H, Hoshi H: Consideration of optimal isodose surface selection for target coverage in micro-multileaf collimator-based stereotactic radiotherapy for large cystic brain metastases: comparison of $90 \%, 80 \%$ and $70 \%$ isodose surface-based planning. Br J Radiol 85:e640e646, 2012

23. Paddick I: A simple scoring ratio to index the conformity of radiosurgical treatment plans. Technical note. J Neurosurg 93 (Suppl 3):219-222, 2000

24. Paddick I, Lippitz B: A simple dose gradient measurement tool to complement the conformity index. J Neurosurg 105 Suppl:194-201, 2006

25. Park JM, Park SY, Ye SJ, Kim JH, Carlson J, Wu HG: New conformity indices based on the calculation of distances between the target volume and the volume of reference isodose. Br J Radiol 87:20140342, 2014

26. Sheehan JP, Pouratian N, Steiner L, Laws ER, Vance ML: Gamma Knife surgery for pituitary adenomas: factors related to radiological and endocrine outcomes. J Neurosurg 114:303-309, 2011

27. Sneed PK, Mendez J, Vemer-van den Hoek JGM, Seymour ZA, Ma L, Molinaro AM, et al: Adverse radiation effect after stereotactic radiosurgery for brain metastases: incidence, time course, and risk factors. J Neurosurg 123:373-386, 2015

28. Sung K, Choi YE: Dose gradient curve: a new tool for evaluating dose gradient. PLoS One 13:e0196664, 2018

29. Tanyi JA, Doss EJ, Kato CM, Monaco DL, Meng LZ, Chen Y, et al: Dynamic conformal arc cranial stereotactic radiosurgery: implications of multileaf collimator margin on dosevolume metrics. Br J Radiol 85:e1058-e1066, 2012

30. Thomas EM, Popple RA, Wu X, Clark GM, Markert JM, Guthrie BL, et al: Comparison of plan quality and delivery time between volumetric arc therapy (RapidArc) and Gamma Knife radiosurgery for multiple cranial metastases. Neurosurgery 75:409-418, 2014

31. Wachter S, Gerstner N, Goldner G, Pötzi R, Wambersie A, Pötter R: Rectal sequelae after conformal radiotherapy of prostate cancer: dose-volume histograms as predictive factors. Radiother Oncol 59:65-70, 2001

32. Wagner TH, Bova FJ, Friedman WA, Buatti JM, Bouchet LG, Meeks SL: A simple and reliable index for scoring rival stereotactic radiosurgery plans. Int J Radiat Oncol Biol Phys 57:1141-1149, 2003

33. Zaider M, Amols HII: Practical considerations in using calculated healthy-tissue complication probabilities for treatment-plan optimization. Int J Radiat Oncol Biol Phys 44:439-447, 1999

34. Zhao B, Jin JY, Wen N, Huang Y, Siddiqui MS, Chetty IJ, et al: Prescription to 50-75\% isodose line may be optimum for linear accelerator based radiosurgery of cranial lesions. J Radiosurg SBRT 3:139-147, 2014

\section{Disclosures}

Mr. Paddick works as an ad hoc consultant for Elekta AB, Stockholm.

\section{Author Contributions}

Conception and design: both authors. Acquisition of data: Dimitriadis. Analysis and interpretation of data: Dimitriadis. Drafting the article: Dimitriadis. Critically revising the article: Dimitriadis. Reviewed submitted version of manuscript: both authors. Approved the final version of the manuscript on behalf of both authors: Dimitriadis. Study supervision: Paddick.

\section{Supplemental Information \\ Previous Presentations}

Part of this work was presented in keynote presentations at the International Stereotactic Radiosurgery Society Congress held in Montreux, Switzerland, on June 1, 2017, and the 19th Leksell Gamma Knife Society Meeting held in Dubai, United Arab Emirates, on March 4-8, 2018.

\section{Correspondence}

Alexis Dimitriadis: Queen Square Radiosurgery Centre, National Hospital for Neurology and Neurosurgery, London, United Kingdom.alex@physicsconsulting.co.uk. 